\title{
XV. On the combination of the theorems of Maclaurin and Taylor
}

\section{J.R. Young}

To cite this article: J.R. Young (1848) XV. On the combination of the theorems of Maclaurin and Taylor, Philosophical Magazine Series 3, 32:213, 98-100, DOI: 10.1080/14786444808645936

To link to this article: http://dx.doi.org/10.1080/14786444808645936

曲 Published online: 30 Apr 2009.

Submit your article to this journal ๘

Џ Article views: 3

Q View related articles $\sqsubset$ 
If the blue radiation or white light liberates iodine or bromine, these elements would evaporate or combine with the silver surface immediately beneath. If we take the first idea, how comes it that the red radiation re-establishes the compound in its primitive proportions: and, in the second case, how does it happen that these rays are capable of decomposing the surface beneath, liberating the iodine or bromine, and then combining them again with the upper surface? It is impossible to admit that the red radiation is endowed at the same time with the property of separating and the property of reuniting the same elements. We must then attribute it to a particular force-electricity perhaps, which might accompany each radiation, and which, under the influence of the one, would act positively, and negatively under the other, without changing the chemical compound. In one case this influence would give the affinity for mercury, and in the other destroy it.

At all events, we must look for another explanation of the phænomenon than the one which has hitherto been received, viz. the decomposition of the iodide of silver by the action of light. It is true that light decomposes iodide of silver, forming a subiodide, but this seems to require a longer time than that during which the surface is endowed with the property of attracting the vapours of mercury. In fact, the last property is communicated nearly instantaneously, which is not the case for the decomposition of the iodide by the action of light.

$\mathrm{XV}$. On the Combination of the Theorems of Maclaurin and Taylor. By J. R. Young, Professor of Mathematics in Belfast College*.

I $N$ the application of Maclaurin's theorem to the develop1 ment of a function $\mathrm{F}(a+x)$ in a series proceeding according to the powers of $x$, we are alway's directed to differentiate the function $\mathrm{F}$ in reference to $x$, and then to put zero for $x$ in the several results. There is an unnecessary expenditure of symbolical work, and therefore of time in following this direction; for it is an axiomatic principle, which $I$ have elsewhere announced $\dagger$, that whether we differentiate $\mathrm{F}(a+x)$ in reference to $x$, and afterwards make $x$ zero, or make $x$ zero first, and then differentiate in reference to $a$, the results are identical; that is,

$$
\left[\frac{d^{n} \mathrm{~F}(a+x)}{d x^{n}}\right]=\frac{d^{n} \mathbf{F}(a)}{d a^{n}}
$$

- Communicated by the Author.

† Mechanics' Magazine, Jan. 15, 1848. 
the brackets indicating what the expression between them becomes when $x=0$.

In writing down Maclaurin's theorem, authors differ considerably as to the notation employed for the coefficients. I have thought it would be well, for the sake of uniformity in this respect, if the plan generally adopted for distinguishing the coefficients of Bernoulli, and which, indeed, are only those of Maclaurin in a particular case, were universally followed. Maclaurin's theorem would thus be written

$$
\mathrm{F}(\alpha+x)=\mathrm{M}_{0}+\mathrm{M}_{1} x+\mathrm{M}_{2} \frac{x^{2}}{2}+\mathrm{M}_{3} \frac{x^{3}}{2.3}+\& \mathrm{c} ., .
$$

and T'aylor's,

$$
\mathrm{F}(x+a)=\mathrm{T}_{0}+\mathrm{T}_{1} a+\mathrm{T}_{2} \frac{a^{2}}{2}+\mathrm{T}_{3} \frac{a^{3}}{2.3}+\& \mathrm{c} . .
$$

Applying the first of these theorems to the function $y=\log$ $(a+x)$, in order to illustrate the foregoing principle, we have, making $x=0$,

$$
\begin{gathered}
\log a=\mathrm{M}_{0}, \frac{d y}{d a}=\frac{1}{a}=\mathrm{M}_{1}, \frac{d^{2} y}{d a^{2}}=-\frac{1}{a^{2}}=\mathrm{M}_{2}, \\
\frac{d^{3} y}{d a^{3}}=\frac{2}{a^{3}}=\mathrm{M}_{3}, \frac{d^{4} y}{d a^{4}}=-\frac{2.3}{a^{4}}=\mathrm{M}_{4}, \\
\& \mathrm{c} . \\
\therefore \log (a+x)=\log a+\frac{x}{a}-\frac{x^{2}}{2 a^{2}}+\frac{x^{3}}{3 a^{3}}-\frac{x^{4}}{4 a^{4}}+\& \mathrm{c} .
\end{gathered}
$$

And, from the process by which the coefficients have here been deduced, it is plain that Taylor's development of log $(x+a)$ is got from this by simply changing the places of $a$ and $x$; and that the one development is always convertible into the other, by thus interchanging the two terms of the binomial under $F$. There is therefore no necessity for considering (1.) and (2.) as distinct theorems : nor is it correct to do so, since they are both comprehended under the sane form ; for whichever be the term according to whose powers the development is to proceed, the coefficients are always to be derived from the function after that term is made zero. Hence, calling either term, indifferently, $t$; and denoting the differential coefficients derived, as here explained, by $\mathrm{D}_{1}, \mathrm{D}_{2}$, \&c., the following theorem comprehends both (1.) and (2.):

$$
y=\mathrm{D}_{0}+\mathrm{D}_{1} t+\mathrm{D}_{2} \frac{t^{2}}{2}+\mathrm{D}_{3} \frac{t^{3}}{2.3}+\& \mathrm{c} \text {. }
$$

Even when the function is not that of a binomial, but of a simple monomial, as $a^{x}, \log x$, \&c., still it will be advisable, in $\mathrm{H} 2$ 
seeking the development, to take the more general form $a^{x+h}$, $\log (x+h), \& c .$, and to proceed as above, afterwards making $h=0$; for we shall thus always be led to true developments, and thence to the cases in which the sought developments are impossible, these being indicated by the hypothesis of $h=0$ causing the coefficients to become infinite. And it is obvious, from the method above described, that the coefficients of the development of $\mathrm{F}(\boldsymbol{x}+h)$ are just as readily obtained as those derived from $\mathrm{F}(x)$ in the usual way.

In thus recommending that $x$ be replaced by $x+h$, when the development of $\mathrm{F}(x)$ is required in powers of $x$, it will be observed, from what is said above, that it is merely proposed to deduce $\mathrm{D}_{0}, \mathrm{D}_{1}, \mathrm{D}_{2}$, \&c. from $\mathrm{F}(h)$. If these are infinite when $h=0$, the inference will be, that, with finite coefficients, the development is impossible. But if we leave $h$ undetermined, and put, in the series, $x-h$ for $x$, we shall then get the development of $\mathrm{F}(x)$, with finite coefficients, in powers of $x-h$; and as $h$ is arbitrary, we may thus approach as near to the proposed development as we please. And although for the purposes of actual numerical computation, the coefficients would become more and more unmanageable, from their increasing magnitude, as $h$ approaches to zero, yet even in this extreme case-in which the coefficients actually become infinite-the development, regarded as the last of the series of developments here spoken of, will still be analytically true.

Belfast, Jan. 5, 1848.

XVI. On the Production of Light by Chemical Action. By John William Draper, M.D., Professor of Chemistry in the University of New York*.

THE production of light and heat by the combustion of various bodies is, of all chemical processes, that which ministers most to the comfort and well-being of man. By it the rigour of winter is moderated, and night made almost as available for our purposes as the day.

One would suppose that, of a phænomenon on which so much of our personal and social happiness depends, and which must have been daily witnessed by every man that has ever lived, all the particulars ought to have been long ago known. Among scientific men its importance has been universally recognized. The early theories of chemistry, such as those of Stahl and Lavoisier, are essentially theories of combustion.

It is nevertheless remarkable how little positive knowledge we still possess on this subject. Some chemists believe that

- Communicated by the Author. 